\title{
Study of fragment in different shapes oblique penetration on circular tube
}

\author{
Xuefei Fan, Weibing $\mathrm{Li}^{\mathrm{a}}$, Guili Zhu and Xiaoming Wang \\ ZNDY of Ministerial Key Laboratory Nanjing University of Science and Technology, Nanjing 210094, China
}

\begin{abstract}
LS-DYNA finite element software was used to set up the computational model of fragment oblique penetration on circular tube, the dynamic process of cylindrical, spherical and cubic fragment penetration on circular tube was simulated under different working conditions, acquired the damage characteristics and ultimate penetration rate of circular tube under the penetration of fragments in different shapes, studied the influencing law between the initial velocity of fragment with the critical angle of ricochet. The results show that the penetration ability of cylindrical fragment is the strongest, the critical damage pattern of circular tube is shear perforation or penetrating cracks; The ultimate penetration rate $\mathrm{Vu}$ and the entry angle $\varphi$ is exponential correlation, the influence of $\varphi$ for Vu mainly embodied in the stage of $\varphi>40^{\circ}$; The initial velocity $\mathrm{V} 0$ has a great influence on the critical angle of ricochet $\varphi \mathrm{u}$ when $\mathrm{Vu}<$ $900 \mathrm{~m} / \mathrm{s}$, the value of $\varphi u$ correspond to the cylindrical fragment in the biggest, the penetration is the most stable; The value of $\varphi \mathrm{u}$ will tend to be a certain value along the V0 increases to $1200 \mathrm{~m} / \mathrm{s}$.
\end{abstract}

Keywords: preformed fragment; circular tube; oblique penetration; ultimate penetration rate; numerical simulation.

\section{Introduction}

The preformed fragment warhead can produce fragment of homogeneous quality and regular shapes, the combination of fragments in different shapes, sizes and arrangement can produce different damage effects for target. So, the study about the penetration efficiency of fragment has always been valued by domestic and foreign scholars, S.S.MI[1,2] studied the penetration law of fragments in different shapes penetration on aluminum alloy target, revealed the influence of fragment shapes and angle of incidence for penetration effect; J.F.WEI[3] studied the penetration law of tungsten alloy cylindrical fragment for multi layer aluminum alloy spacer target by experiment, the empirical formula of calculating target plate numbers was acquired; N.ZHOU[4] analyzed the damage mechanism and failure mode of composite target under the action of different shapes fragment by ballistic gun experiment. The above works can provide some references for the design of preformed fragment warhead, the above researches are mainly aimed at the homogeneous target plate or composite planar target plate, for the study of cylindrical target(the shell of missile cabin, the cabin of submarine and ship, aircraft skin and high pressure gas storage tank, gas transmission pipeline of armored vehicle, warship, aircraft and so on) lack of attentions. Brorvik. T[5], Nishida. M[6], J..MU[7] studied the

a Corresponding author : njustlwb@163.com 
impact action of projectile for circular tube, analyzed the failure mode of circular tube, but these researches only considered the positive penetration, the intersection of fragment with target can be arbitrary directions in practice.

LS-DYNA finite element software was used to conduct numerical simulation of the dynamic process that cylindrical, spherical and cubic fragment in equivalent quality penetration on tick walled circular tube, studied the damage characteristics of circular tube under the action of different shapes fragment, analyzed the influence of fragment shapes and penetration angle for the ultimate penetration rate of circular tube, found out the corresponding relation of fragment shapes in different penetration speed with the critical angle of ricochet.

\section{Material model and parameters}

Under the action of impact load, the circular tube will generate rheological behavior under the conditions of large deformation, high strain rate and high temperature, Johnson-Cook material model and GRUNISEN state equation is used to describe the dynamic response process[8].

The constitutive equation of Johnson-Cook is:

$$
\sigma_{y}=\left(A+B \bar{\varepsilon}^{p^{n}}\right)\left(1+C \ln \varepsilon^{*}\right)\left(1-T^{* m}\right) \quad\left(T^{*}=\frac{T-T_{\text {room }}}{T_{\text {melt }}-T_{\text {room }}}\right)
$$

In the equation: $\sigma_{y}$ is the material flowing yield strength, $\mathrm{A}$ is yield stress, $\mathrm{B}$ is strain hardening coefficient, $\mathrm{n}$ is strain hardening exponent, $\mathrm{C}$ is strain rate coefficient, $\bar{\varepsilon}^{p}$ is equivalent plastic strain, $\varepsilon^{*}$ is equivalent plastic strain rate, $\mathrm{m}$ is temperature coefficient. $T^{*}$ is non dimensional temperature, $\mathrm{T}$ is material temperature, $T_{\text {melt }}$ is material melting temperature, $T_{\text {room }}$ is room temperature, the material parameters in Table 1 are used for circular tube and preformed fragment.

Table 1. Material parameters

\begin{tabular}{llllllllll}
\hline Materials & $\begin{array}{l}\mathrm{A} \\
(\mathrm{MPa})\end{array}$ & $\begin{array}{l}\mathrm{B} \\
(\mathrm{MPa})\end{array}$ & $\mathrm{n}$ & $\mathrm{c}$ & $\mathrm{m}$ & $\begin{array}{l}T_{\text {room }} \\
(\mathrm{K})\end{array}$ & $\begin{array}{l}T_{\text {melt }} \\
(\mathrm{K})\end{array}$ & $\begin{array}{l}\rho \\
\left(\mathrm{g} / \mathrm{cm}^{3}\right)\end{array}$ & $\begin{array}{l}c_{p} \\
(\mathrm{~J} / \mathrm{Kg} . \mathrm{K})\end{array}$ \\
\hline $\begin{array}{l}\text { 45\#steel } \\
\begin{array}{l}\text { Tungsten } \\
\text { Alloy }\end{array}\end{array}$ & 496 & 434 & 0.307 & 0.0084 & 0.804 & 300 & 1793 & 7.83 & 477 \\
\hline
\end{tabular}

The pressure expression of GRUNEISEN state equation is:

$$
P=\frac{\rho_{0} C^{2} \mu\left[1+\left(1-\frac{\gamma_{0}}{2}\right) \mu-\frac{\alpha}{2} \mu^{2}\right]}{\left[1-\left(S_{1}-1\right) \mu-S_{2} \frac{\mu^{2}}{\mu+1}-S_{3} \frac{\mu^{3}}{(\mu+1)^{2}}\right]^{2}}+\left(\gamma_{0}+\alpha \mu\right) E
$$

$\rho_{0}$ is the initial material density, $\mathrm{E}$ is internal energy, $\rho$ is material density corresponding to the current time step, $C, S_{1}, S_{2}, S_{3}, \gamma_{0}, \alpha$ is material characteristic parameters, $\mu=\rho / \rho_{0}-1$.

The values of material parameters in the finite element model are: for $45 \#$ steel, $C=0.4569 \mathrm{~cm} / \mathrm{uS}, S_{1}=1.49, \gamma_{0}=2.17, \alpha=0.46$; for tungsten alloy, $C=0.399 \mathrm{~cm} / \mathrm{us}, S_{1}=1.24$, $\gamma_{0}=1.54$.

\section{The establishment of finite element model}

Preformed fragments can act on a point in the circular tube along any direction, Figure 1 is a schematic diagram of preformed fragment penetrating circular tube, the plane which through the 
center line of the circular tube and penetration point is called axial angle, the plane which through the cross section of circular tube and penetration point is called circumferential plane[9], the included angle of penetration direction with penetration point's normal is entry angle $\varphi$. This paper studied the situation of preformed fragment penetration on circular tube along a certain direction in axial or circumferential plane.

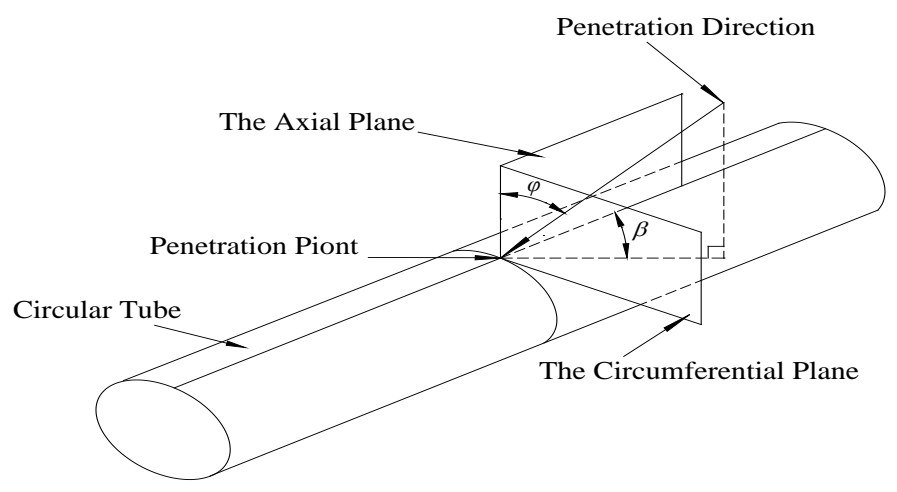

Figure 1. Schematics Diagram

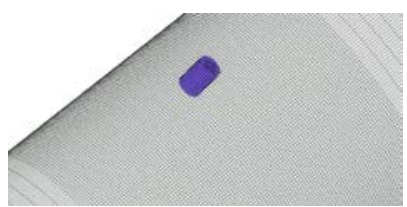

Figure 2. Grid Encryption

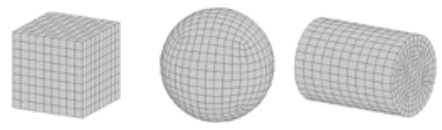

Figure 3. The Model of Fragment

The object which studied in this paper is a circular tube fixed at both ends, the length is $800 \mathrm{~mm}$, the external diameter is $50 \mathrm{~mm}$, the wall thickness is $9 \mathrm{~mm}$ and the material is $45 \#$ steel, the diameter and length of the cylindrical preformed fragment is $10.2 \mathrm{~mm}$, the length of the cubic fragment is $8.2 \mathrm{~mm}$, the material of fragment is tungsten alloy, the quality of these three fragment is $9.7 \mathrm{~g}$. In order to improve the accuracy of numerical simulation, the whole model is established to conduct numerical analysis, encrypt the grid in contact area (as shown in Fig.2), the finite element model of fragment is shown in Fig.3. Lagrange algorithm is used in circular tube and fragment.

\section{Simulation results and analysis}

The above finite element model is used, the entry angle $\varphi$ of fragment in the model is changed, the response characteristics and law of circular tube under the action of oblique penetration are studied. The positive penetration on circular tube and the entry angle respectively take $10^{\circ}, 20^{\circ}, 30^{\circ}, 40^{\circ}, 50^{\circ}$, $60^{\circ}$ in axial or circumferential plane are calculated, a total of 39 working conditions, used for analysis the penetration ability of preformed fragment and the damage characteristics of circular tube, the influence of entry angle $\varphi$ for the ultimate penetration rate and the critical angle of ricochet.

\subsection{The penetration ability of different shapes fragments and the damage characteristics of circular tube}

The shape of preformed fragment is the important factor for the final power of warhead, the effect of fragment for circular tube target are mainly embodied in the penetration damage caused by collision, Fig.4 shows the velocity variation of these three preformed fragment in the penetration process when initial velocity is $900 \mathrm{~m} / \mathrm{s}$. 

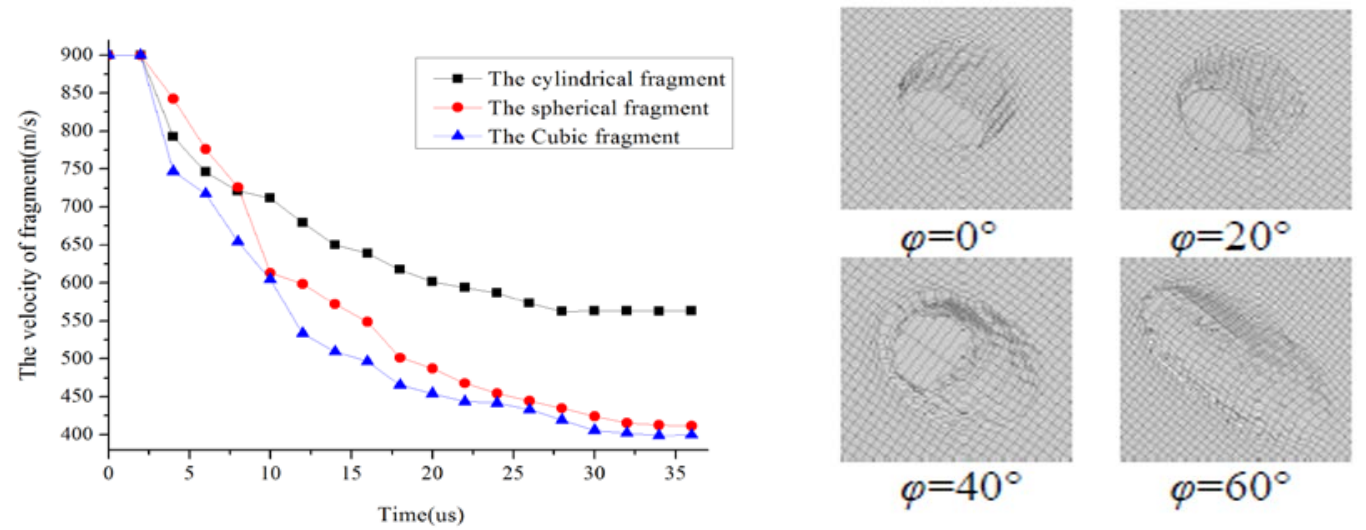

Figure 4. The Velocity Variation of Fragments

Figure 5. The Damage Mode of Circular Tube

Analysis the changing law of fragment velocity, at the initial stage(3-10 $\mu \mathrm{s})$ of fragment penetration on circular tube, the impedance force that circular tube applied to the frament is the biggest, the velocity attenuation is very rapid, as the penetration process continue, the ennergy dissipation of fragment will cause the interating force between circular tube with fragment decrease, the velocity attenuation trend of fragment gradually slow down. The curve in the diagram are compared, the cylindrical fragment compelets penetration at $28 \mu \mathrm{s}$, the residual velocity is $563 \mathrm{~m} / \mathrm{s}$; The spherical and cubic fragment compelet penetration at $32 \mu \mathrm{s}$, the residual velocity respectively are $412 \mathrm{~m} / \mathrm{s}$ and $398 \mathrm{~m} / \mathrm{s}$. The penetration ability of cylindrical fragment is the strongest, the spherical fragment takes second place and the cubic fragment is the weakest by comparing.

Under the condition of a certain size of circular tube and dragment, when the material is certain, the damage model of circular tube is related to the shapes, entry velocity and entry angle of fragment, at the basic of researched te penetration ability of different shapes fragment, the cirtical damage characteristics of circular tube under the impact of these three shapes fragments in different entry angle are acquired by simulating.

The deformation mode of circular tube under the local impact contains local sag deformation, whole bending deformation and the coupling of them, adiabatic shear will take place and form a plug damage in the circular tube because of it's large rigidity.

Fig.5 shows the critical damage mode of circular tube under the oblique penetration of cylindrical fragment in axial plane, when the inclination angle of fragment $\varphi$ within $20^{\circ}$, the penetration phenomenon closes to the positive penetration; When $\varphi>30^{\circ}$, the moment caused by target impedance force will gradually increases and it will lead to fragment rotation, the fragment will overturn and the penetration path will change, the fragment maybe turn to the positive direction or ricochet, the fragment turn to a positive direction and penetrate circular tube when $\varphi=40^{\circ}$, the fragment generate penetrating cracks on the surface of circular tube. The further study find that the critical angle of generating cracks $\varphi=55^{\circ}$, the circular tube has the same damage mechanism under the action of spherical and cubic fragment, the entry angle $\varphi$ that generate penetrating cracks within between $50^{\circ}$ with $55^{\circ}$, this shows that the spherical and cubic fragment will ricochet easily.

\subsection{The influence of entry angle for the ultimate penetration rate}

The ultimate penetration rate is the minimum speed that preformed fragment penetrate the circular tube or generate penetrating cracks on the surface of circular tube, it is an important indicator to measure the penetration resistance of circular tube. On the basis of 3.1 studies, the ultimate penetration rate of these three fragments in different entry angle penetration on circular tube in axial or circumferential plane is acquired by simulating, as shown in Fig.6.

Conduct the curve fitting of the data in (a) and (b) of Fig.6, the changing law of the ultimate 
penetration velocity $\mathrm{Vu}$ along the change of entry angle $\varphi$ is acquired.

The axial penetration is:

$$
V_{u}= \begin{cases}537.9+99.4 e^{\varphi / 51.93}+0.9 e^{\varphi / 9.63} & \text { Cylindrical } \\ 1107.2-477.0 e^{(\varphi-41.8) / 43}+247.1 e^{(\varphi-41.8) / 33} & \text { Spherical } \\ 725.3+11.0 e^{\varphi / 13.8} & \text { Cubic }\end{cases}
$$

The circumferential penetration is:

$$
V_{u}= \begin{cases}799.6-685.6 e^{\varphi / 36.1}+535.9 e^{\varphi / 28.80} & \text { Cylindrical } \\ 1270.3-1081.8 e^{\varphi / 54.2}+542.1 e^{\varphi / 31.0} & \text { Spherical } \\ 1134.1+399.0 e^{\varphi / 29.8}-792 e^{\varphi / 54.4} & \text { Cubic }\end{cases}
$$

From the above rules, the ultimate penetration rate $\mathrm{Vu}$ presents exponential growth along the increases of entry angle $\varphi$ under these two penetration modes. The increasing amplitude is a little of small when $\varphi<40^{\circ}$, this is because the fragment under the action of impedance moment overturn to internal when entry angle is small. When $\varphi>40^{\circ}$, the flip moment that circular tube applied to fragment make it deviate from the axis of circular tube, the penetration path become longer until fly from the surface of the circular tube, in this stage, the ultimate rate Vu rises rapidly along the increases of entry angle $\varphi$.

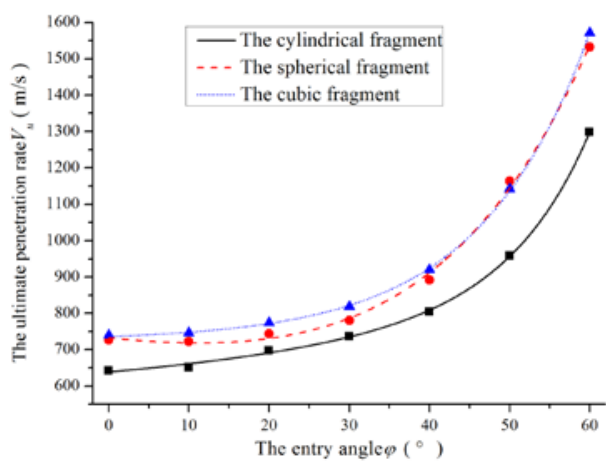

(a) The Axial Oblique Penetration

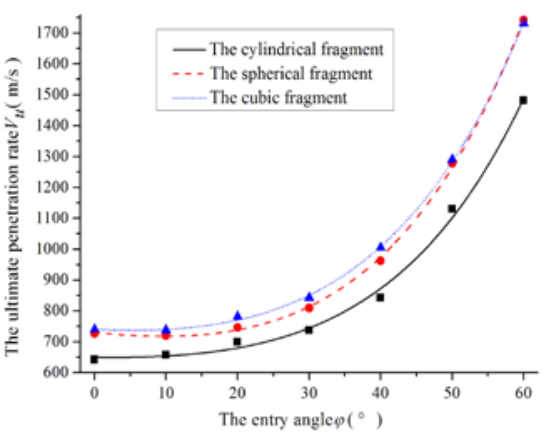

(b) The Circumferential Oblique Penetration

Figure 6. The Relation Curve of the Ultimate Penetration Rate with Entry Angle

The ultimate penetration rate of cylindrical fragments is less than the ultimate penetration rate of spherical and cubic fragment, When $\varphi<40^{\circ}$, the ultimate penetration rate of spherical fragment is less than the ultimate penetration rate of cubic fragment, when $\varphi>40^{\circ}$, the ultimate penetration rate of spherical and cubic fragment are the same basically, the spherical and cubic fragment have the same penetration ability when $\varphi>40^{\circ}$. In addition, the ultimate penetration rate of axial penetration is larger than the values of circumferential penetration, this shows that the circular tube is more easily damaged under the action of axial oblique penetration.

\subsection{The critical angle of ricochet of fragment in different initial velocity}

The fragment may penetrate , embed and ricochet in the penetration process, the penetration refers to the fragment pass through the circular tube; Embedding is the phenomenon that fragments are prevented by circular tube and stay in the circular tube; The ricochet is the phenomenon that the 
fragment jump from the surface of circular tube and continue to move in the air after it impact or penetrate on the circular tube, the main factors to ricochet are the entry angle of fragment and the energy or structure of fragment. Aimed at cylindrical, spherical and cubic fragment penetration on circular tube, the critical angle of ricochet of fragment in different initial velocity in axial or circumferential plane are acquired by simulating.

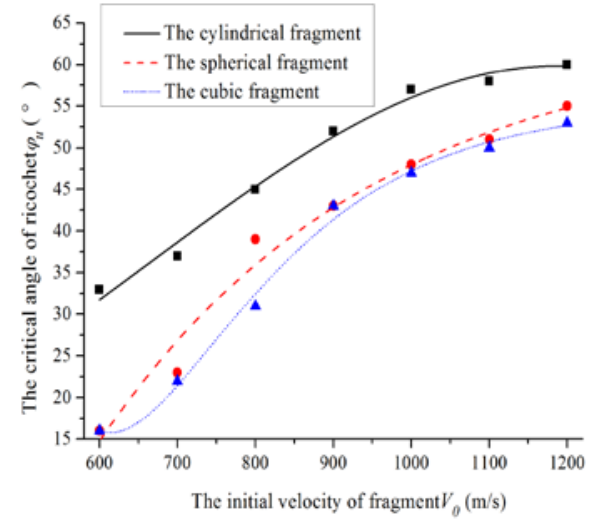

(a) The Axial Oblique Penetration

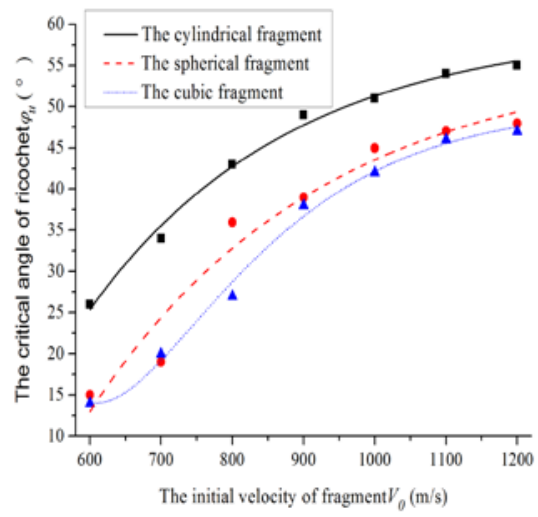

(b) The Circumferential Oblique Penetration

Figure 7. The Relation Curve of the Critical Angle of Ricochet with the initial Velocity of Fragment

The curve in (a) and (b) of Fig.7 are fitted:

The axial oblique penetration:

$$
\varphi_{u}= \begin{cases}e^{1.5+0.0043 V_{0}-1.82 \times 10^{-6} V_{0}^{2}} & \text { Cylindrical } \\ 63.7-268.6 e^{-V_{0} / 352.5} & \text { Spherical } \\ 55+8.5 e^{-\left(V_{0}-1175\right) / 124}-11 e^{-\left(V_{0}-1175\right) / 130} & \text { Cubic }\end{cases}
$$

The circumferential oblique penetration:

$$
\varphi_{u}= \begin{cases}59.6-285.5 e^{-V_{0} / 283} & \text { Cylindrical } \\ 56.2-23 e^{-\left(V_{0}-805\right) / 326} & \text { Spherical } \\ 50+5.3 e^{-\left(V_{0}-1313\right) / 134}-6.6 e^{-\left(V_{0}-1313\right) / 138} & \text { Cubic }\end{cases}
$$

The critical angle of ricochet $\varphi \mathrm{u}$ presents exponential change with the initial velocity $\mathrm{V} 0$, when V0< $900 \mathrm{~m} / \mathrm{s}$, $\varphi \mathrm{u}$ substantially increases along the increases of V0, V0 has a great influence for $\varphi \mathrm{u}$ at low speed stage; When V0>900m/s, the changing amplitude of $\varphi$ u decreases with V0, the bigger the V0, the smaller the changing amplitude of $\varphi \mathrm{u}$. The critical angle of ricochet $\varphi \mathrm{u}$ and changing rules of spherical and cubic fragment are similar by comparing, the critical angle of ricochet $\varphi u$ of cylindrical fragment is bigger than previous both, this shows that the cylindrical fragment is not easily happen ricochet.

From the rules of curve in Fig.7, the curve tends to horizontal when the speed increases to $1200 \mathrm{~m} / \mathrm{s}$, the value of $\varphi \mathrm{u}$ will tend to be a certain value, the $\varphi \mathrm{u}$ of cylindrical fragment will tend to $60^{\circ}$, when axial oblique penetration, the values of spherical and cubic fragment will settle in $50^{\circ}$; The $\varphi \mathrm{u}$ of spherical and cubic fragment will tend to $55^{\circ}$ when circumferential oblique penetration, the $\varphi \mathrm{u}$ of spherical and cubic fragment will settle in $48^{\circ}$, so the circumferential oblique penetration is more easily to ricochet. 


\section{Conclusions}

LS-DYNA finite element software is used to simulate the dynamic process of preformed fragment penetration on circular tube, the penetration ability of different shapes fragments and the damage characteristics of circular tube are studied, the changing rules of the ultimate penetration rate with the critical angle of ricochet are analyzed, the following conclusions are drawn:

(1) The penetration ability of cylindrical fragment is the strongest, the spherical fragment takes second place, and the cubic fragment is the weakest. The critical damage mode of circular tube under the oblique penetration of preformed fragment mainly are shear perforation and penetrating cracks, when $\varphi \leq 30^{\circ}$, it is straight punch, when $30^{\circ}<\varphi \leq 50^{\circ}$, the penetration path will deflect, when $\varphi>50^{\circ}$, it will generate penetrating cracks.

(2) Vu presents exponential correlation with $\varphi$, but the influence of $\varphi$ for Vu mainly embodied in the stage of $\varphi>40^{\circ}$; The values of $\mathrm{Vu}$ correspond to cylindrical fragment are smaller than the $\mathrm{Vu}$ of spherical and cubic fragment, when $\varphi>40^{\circ}$, the $\mathrm{Vu}$ of spherical and cubic fragment are basically the same; The circular tube is more easily damaged under the action of axial oblique penetration.

(3) When $\mathrm{V} 0<900 \mathrm{~m} / \mathrm{s}$, it has a great influence for $\varphi \mathrm{u}$, $\varphi \mathrm{u}$ presents exponential correlation with V0; The $\varphi \mathrm{u}$ of spherical and cubic fragment are close and the laws are similar, the $\varphi \mathrm{u}$ of cylindrical fragment is the biggest, the penetration is the most stable; The value of $\varphi$ u will tend to be certain along the increases of $\mathrm{V} 0$, the circumferential oblique penetration is more easily ricochet.

\section{Acknowledgements}

The work presented in this paper has been funded by the National Natural Science Foundation of China, and sponsored by Qing Lan Project.

\section{Reference}

1. S.S.Mi, X.E.ZHANG, G.M.TAO. Finite element analysis of spherical fragments penetrating LY-12 aluminum alloy target, J. Explosions and Shock Waves, 25(2005)477-480.

2. T.LI, S.S.MI, W.T.JIN. Numerical simulation research of the penetration performance of several kinds of steel fragments, J. Journal of Projectiles, Rockets, Missiles and Guidance, 29(2009)130-133.

3. J.F.WEI, S.S.WANG, F.MA. Experimental investigation on cylindrical fragments penetrating into multi-spaced targets, J. Journal of Projectiles, Rockets, Missiles and Guidance, 29(2009)123-124.

4. N.ZHOU, J.X.WANG, J.XIE, et al. Experimental study on influence of fragment shape on the anti-penetration performance of composite plates, J. Journal of Ballistics, 26(2014)95-99.

5. T. Borvik, M. Langseth, S. Hopperstad, et al. Perforation of $12 \mathrm{~mm}$ thick steel plates by $20 \mathrm{~mm}$ diameter projectiles with flat, hemisgpherical and conical noses, Part I:experimental study , J. International Journal of Impact Engineering, 27(2002)19-35.

6. M. Nishida, K. Tanaka. Experimental study of perforation and cracking of water-filled aluminum tubes impacted by steel spheres, J. International Journal of Impact Engineering, 32(2006)2000-2016.

7. J.C.MU,T.G.ZHANG. Rupture of this metal tubes by normal and oblique impact of blunt conical-nosed missiles: experiments, J. Acta Mechanica Solida Sinica, 21(2000)49-56.

8. X.S.DAI, J.M.MA. Energy absorbed by a metal tube under axial crush load, J. Journal of Vibration and Shock, 31(2012)100-103.

9. M.WANG, D.W.HUANG, J.H.QU, et al. Simulation on the deformation and fracture of long-rod projectile of tungsten alloy penetrating into 45\#steel, J. Journal of Plasticity Engineering, 19(2012)102-106. 